
\title{
Research S Surate \\ Plasmonic random laser biosensor on fiber facet for label-free detecting biomolecules
}

\author{
Xiaoyu Shi \\ Beijing University of Technology \\ Kun Ge \\ Beijing University of Technology \\ Junhua Tong \\ Beijing University of Technology \\ Tianrui Zhai ( $\nabla$ trzhai@bjut.edu.cn ) \\ https://orcid.org/0000-0003-1922-2386
}

\section{Research}

Keywords: random lasers, plasmonic, fiber facet, polymer, biosensor

Posted Date: February 17th, 2020

DOI: https://doi.org/10.21203/rs.2.23635/v1

License: (c) (i) This work is licensed under a Creative Commons Attribution 4.0 International License. Read Full License 


\title{
Plasmonic random laser biosensor on fiber facet for label-free detecting biomolecules
}

\author{
Xiaoyu Shi, Kun Ge, Junhua Tong, Tianrui Zhai* \\ College of Applied Sciences, Beijing University of Technology, Beijing, 100124, China \\ *Email: trzhai@bjut.edu.cn
}

\begin{abstract}
Low-cost and miniaturized biosensors are key factors leading to the possibility of portable and integrated biomedical system, which play an important role in clinical medicine and life sciences. Random lasers with simple structures provide opportunities for detecting biomolecules. Here, a low-cost biosensors on fiber facet for label-free detecting biomolecules is demonstrated resorting to plasmonic random laser. The random laser is achieved resorting to a random plasmonic scattering structure of Ag nanoparticles and polymer film on fiber facet. Refractive index sensitivity and near-surface sensitivity of the biosensor are systematically studied. Furthermore, the biosensor is used to detect $\operatorname{lgG}$ through specific binding to protein A, exhibiting the detecting limit of $0.68 \mathrm{nM}$. It is believed that this work may promote the applications of plasmonic random laser bio-probe in portable or integrated medical diagnostic platforms, and provide fundamental understanding for the life science.
\end{abstract}

Keywords: random lasers, plasmonic, fiber facet, polymer, biosensor 


\section{Background}

The growing requirements of clinical medicine and life sciences spark off a remarkable trend of bio-sensing technology $y^{1,2}$, which provide important insight into pharmacological intervention and cellular processes ${ }^{3}$. Optical biosensor is one of the most widely used biosensors ${ }^{4-6}$. It commonly obtains the information of target biomolecules through detecting changes in optical signals that caused by the molecule interaction ${ }^{7}$. Towards the advanced sensing technology, optical biosensor initially employs labeled biomolecule to extract information about target biomolecules through the spectral characteristics (eg. fluorescence intensity variation or wavelength shift $)^{8,9}$. However, label-based biosensor would weaken the biological activity or interfere the biological interaction. Label-free biosensor without biomarkers effectively avoid these negative factors from labelling process, providing new functionalities and opportunities ${ }^{10}$. As is well known, high sensitive and detection limit play a central role of sensors ${ }^{11}$. Therefore, great efforts are needed to develop new types of label-free biosensor to optimize the sensing performance.

Laser with narrow spectral linewidth and high intensity implemented as label-free biosensors could resolve smaller wavelength shifts, which is associated with a smaller vitiation of analytes ${ }^{12}$. Biological molecules attached to dielectric cavity can change its effective refractive index, leading to the changes of resonant mode. Currently, laser-based label-free biosensors with extremely high detection sensitivity have been achieved resorting to different kinds of cavities, such as whispering gallery mode lasers $^{13}$, distributed feedback lasers ${ }^{14,15}$, photonic crystal lasers ${ }^{16,17}$ and plasmonic 
$\operatorname{lasers}^{18}$. However, label-free biosensing by traditional lasers suffers severely from meticulous designs and precise preparations of the special optical resonator cavities, which are challenging and high-cost for sensing. Therefore, it is extremely desirable to design label-free laser biosensor with characteristics of simplified preparation and low-cost to promote the development of portable and integrated biomedical system.

Random lasers (RL) characterized with simple structure and flexible design, are good candidates for label-free laser biosensor ${ }^{19,20}$. Without rigid and expensive optical cavities, random laser is based on the multiple scattering of light in low-cost disorder system. The lasing characters are highly dependent on the gain ${ }^{21,22}$ and scattering structure $^{23} 24$. Based on this feature, such random lasing sensors have been used to sense of temperature ${ }^{25}$, chemicals ${ }^{26}$, tumor tissue ${ }^{27}$, cell counting ${ }^{28}$, and bone tissue structure $^{29}$. Furthermore, random laser sensors based on optical fiber are proposed ${ }^{30}$, which not only retain the advantages of the random laser sensor, but also make full use of the high optical transmission efficiency of the optical fiber. The compact structure promotes the miniaturization and integration of the random laser biosensors, bringing new opportunities for portable and integrated biomedical sensing system. However, random lasers biosensors based on optical fiber for monitoring biomolecules interactions have not been investigated till now. In view of their potential functions for life sciences and medical diagnosis, biomolecules sensors based on random laser with optical fibers are in a great need to be developed.

Here, a label-free biosensor based on low-cost plasmonic random laser on fiber facet is designed, fabricated, and demonstrated. The random lasers are fabricated by 
covering polymer membrane over the self-assembly silver nanoparticles (Ag NPs) randomly distributed on fiber facet. We have systematically studied the random lasing action and explored their applications in refractive index sensing and near-surface sensitivity, revealing the important feature that the wavelength shift of lasing mode is proportional to the changes in external refractive index of random laser. The biosensing capability of the random laser on fiber facet is demonstrated by detecting human $\operatorname{lgG}$. Through specific binding to protein-A fixed on the surface of random laser, the concentration of $\lg G$ has been well monitored.

\section{Results and discussion}

In our experiment, Ag NPs with local surface plasmon resonance act as the scattering structure of the random laser, which are self-assembled on the fiber facet by polyvinylpyrrolidone (PVP)-assisted reaction ${ }^{26}$, as is shown in Figure 1a and supplementary materials. $\mathrm{AgNO}_{3}(0.2 \mathrm{~g} / \mathrm{ml})$ and PVP $(0.03125 \mathrm{~g} / \mathrm{ml})$ are dissolved in DI water, as the precursor solution. An optical fiber with the diameter of $800 \mu \mathrm{m}$ is dipped in the precursor solution, and be heated at $200{ }^{\circ} \mathrm{C}$ for $0.5 \mathrm{~h}$. The scanning electron microscope (SEM) images of Ag NPs in Figure 1b shows that the spherical Ag NPs are randomly distributed on the fiber facet for providing strong confinement and feedback of light. The statistical distribution of Ag NPs indicates that the diameter is relatively uniform and mainly distributed between $20 \mathrm{~nm}$ and $30 \mathrm{~nm}$ (Figure 1c). The mean diameter of Ag NPs is estimated as $25 \mathrm{~nm}$. The extinction spectrum exhibits a broad peak from $350 \mathrm{~nm}$ to $650 \mathrm{~nm}$ (see in Figure 1d). The normalized electric field distribution near Ag NPs is stimulated. Due to the localized surface 
plasmon resonance (LSPR) of Ag NPs, their local fields are 10 times enhanced (inset of Figure 1d) respected to air, which can enhance the emissions from the random $\operatorname{lasers}^{20,31-33}$.

(a)

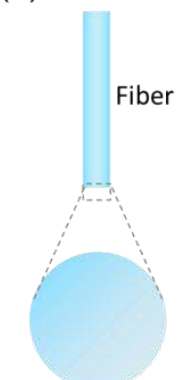

Fiber facet

(c)

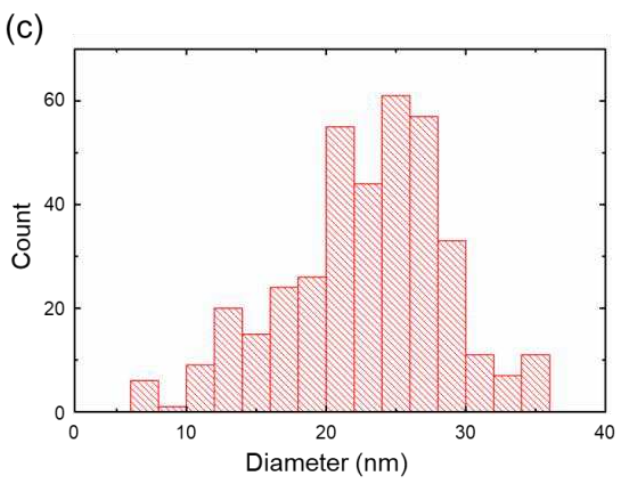

(b)

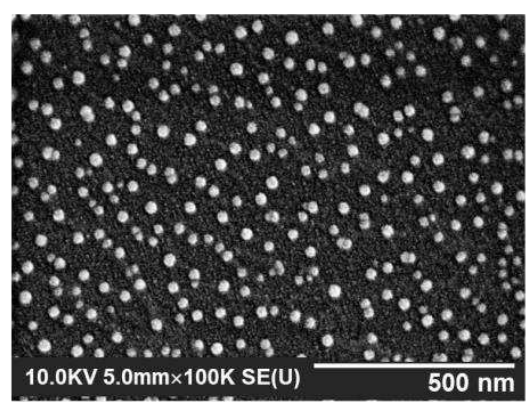

(d)

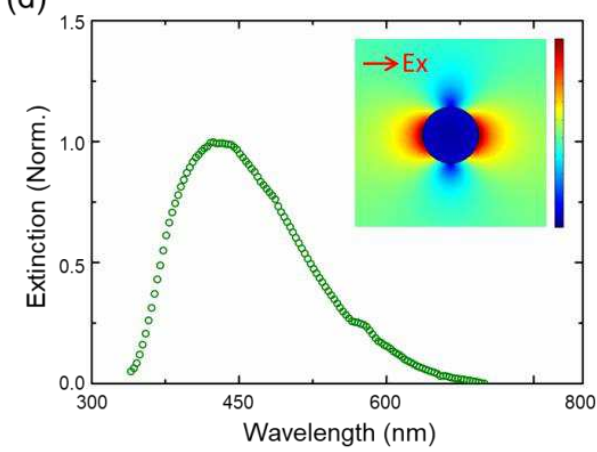

Figure 1 (a) Schematic illustration of the synthesis process for scattering structure on fiber facet.

(b) Scanning electron microscopy (SEM) image of Ag NPs self-assembled on fiber facet. (c)

Statistical distribution of Ag NPs that self-assembled on fiber facet. (d) The extinction spectrum of Ag NPs. Inset: the normalized electric field intensity of Ag NP, the excitation is a plane wave polarized in the $\mathrm{x}$ axis with a wavelength of $466 \mathrm{~nm}$.

Poly[9, 9-dioctylfluorenyl-2, 7-diyl] end capped with DMP (PFO) is an attractive light emitting polymer, characterized with high luminescence efficiency and high charge carrier mobility ${ }^{34}$. Additionally, PFO film with negatively charged surface has been applied in chemical sensors, biological labels and optoelectronic device ${ }^{35-37}$. Thus, PFO chosen as the gain material for the random laser, is transplanted to the 
fiber facet (Figure 2a). Optical micrograph of the front view of the random laser on fiber facet illustrates that the surface of the polymer is uniform and smooth (inset of Figure 2a). The absorption spectrum of PFO displays a absorption band from $320 \mathrm{~nm}$ to $430 \mathrm{~nm}$, which is overlapped with the extinction band of Ag NPs (blue shading) (Figure 2b). It indicates that the excitation of random laser could be enhanced by Ag $\mathrm{NPs}^{38}$. The photoluminescence spectra of PFO with or without Ag NPs are shown in Figure $2 b$, respectively. These two photoluminescence spectra demonstrate that the fluorescence intensity is enhanced by Ag NPs. And the photoluminescence lifetime is further studied by comparing the photoluminescence decay dynamics of PFO with (red dots) and without Ag NPs (blue dots). It can be confirmed that the interaction with $\mathrm{Ag}$ NPs reduced the photoluminescence lifetime from $0.8 \mathrm{~ns}$ to $0.5 \mathrm{~ns}$ (Figure $2 \mathrm{c})^{20}$. In addition, the local field enhancement by Ag NPs has been also been demonstrated by simulating the normalized electric field distribution near Ag NPs that randomly distributed on fiber facet. The result in Figure 2d demonstrates that Ag NPs, especially the Ag NPs dimmer with gap of 5-10 nm, could provide enormous local enhancement and enhance the scattering light from $\mathrm{Ag} \mathrm{NPs}{ }^{39-42}$. As a result, the Ag NPs can supply strong gain and effective feedback for the random lasing. 
(a)

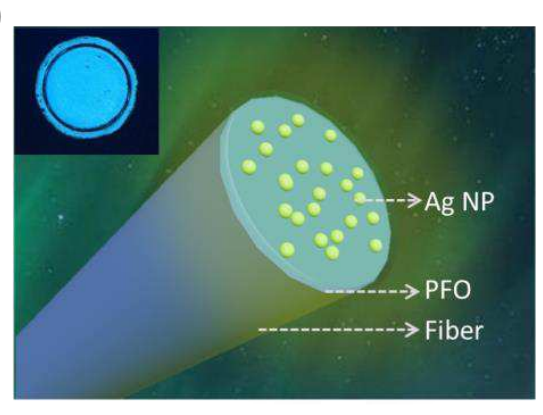

(c)

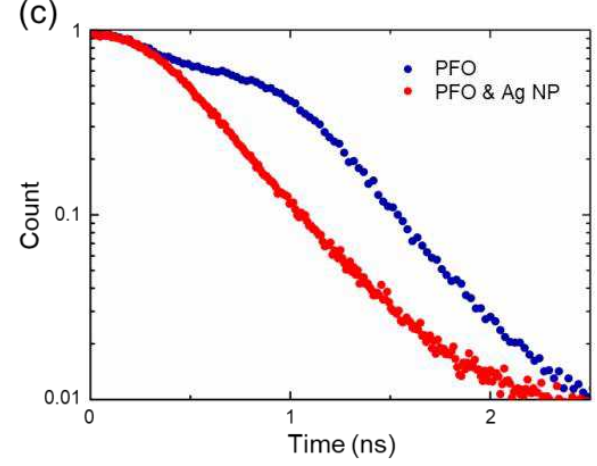

(b)

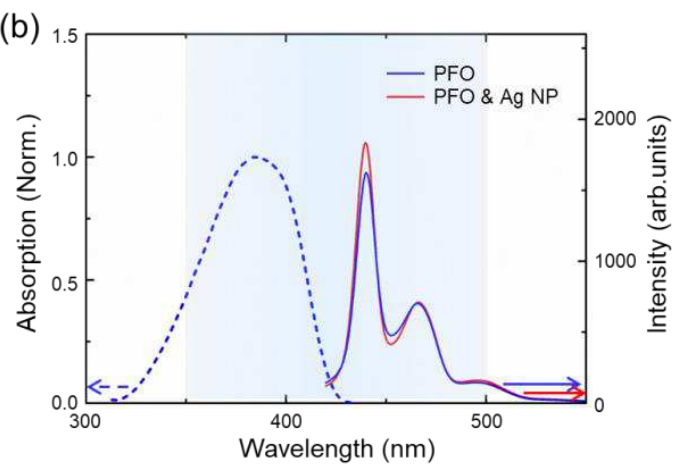

(d)

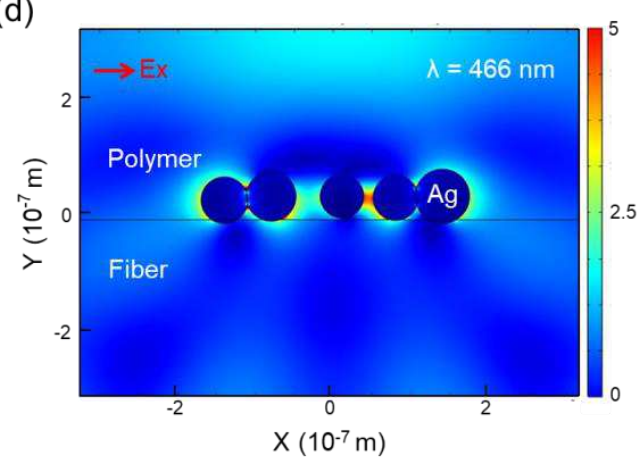

Figure 2 (a) Design of the plasmonic random laser on fiber facet. (b) Absorption spectra (blue dash) and photoluminescence spectra of PFO (blue solid), photoluminescence spectra of polymer with Ag NPs (red solid). (c) The PL lifetime of PFO with (red dots) and without Ag NPs (blue dots). (d) The normalized electric field intensity of Ag NPs on the fiber facet covered by PFO, the excitation is a plane wave polarized in the $\mathrm{x}$ axis with a wavelength of $466 \mathrm{~nm}$.

The emission characteristic of the plasmonic random laser on fiber facet is systematically investigated. Figure 3 a shows the schematic of the experimental setup. The random laser is directly pumped by a pulse laser at $400 \mathrm{~nm}$. The emission is detected at angle $\theta$, which is respect to the orientation vertical to the fiber. Figure $3 \mathrm{~b}$ depicts the evolution of the emission spectra by varying the pump energy densities. When the pump energy density is $19 \mu \mathrm{J} / \mathrm{cm}^{2}$ (red curve in Figure 3b), the spectrum exhibits a broad spontaneous emission band (black curve in Figure 3b). By increasing the pump energy density to $33 \mu \mathrm{J} / \mathrm{cm}^{2}$, the spectrum appears a narrow emission band 
centered at $\lambda=468 \mathrm{~nm}$ with several sharp spikes. It should be noticed that the linewidth of the sharp peaks recorded by a high-resolution spectrometer is as narrow as $0.42 \mathrm{~nm}$ (Figure 3c), indicating the coherent feedback being formed by Ag NPs. As the pump energy density further increases to $39 \mu \mathrm{J} / \mathrm{cm}^{2}$ and $60 \mu \mathrm{J} / \mathrm{cm}^{2}$, the emission intensity increases more rapidly (blue and green curves shown in Figure 3b). Figure $3 \mathrm{~b}$ plots the variation of emission intensity at $468 \mathrm{~nm}$ as a function of the pump energy density. There is a clear threshold behavior with a knee point in the curve as the pump energy densities increase, which further suggests the occurrence of random lasing. According to the threshold curve, the threshold of random lasing is measured at $30.5 \mu \mathrm{J} / \mathrm{cm}^{2}$. The photograph inset of Figure $3 \mathrm{~d}$ shows the bright optical fiber excited by the pump beam on the end of fiber, demonstrating the random laser operating well. Furthermore, spatial emission performance of this plasmonic random laser on fiber facet is studied by changing detection angle from $5^{\circ}$ to $175^{\circ}$ under a fixed pump condition. The integrated intensities of observed spectra in Figure $3 \mathrm{e}$ increase from the sides to the center. As the thickness of the PFO film decreases from the central to the edge of the fiber facet during the film-forming process, the random laser performs an optimal spatial distribution ${ }^{43}$. 
(a)

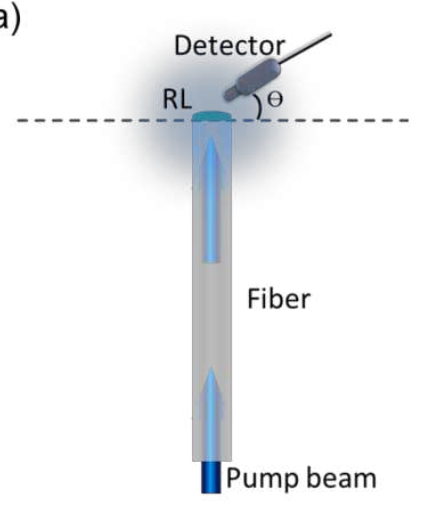

(d)

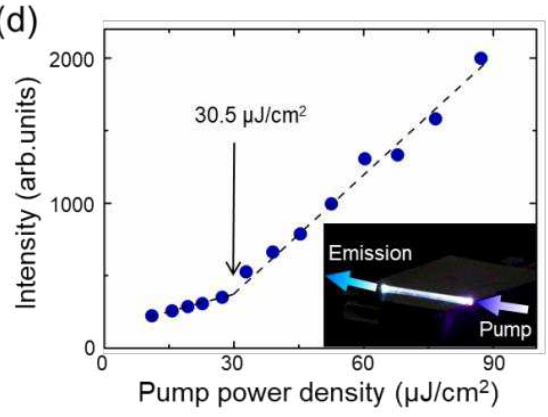

(b)

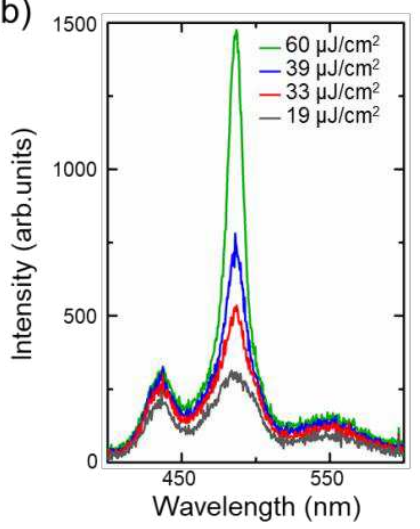

(c)

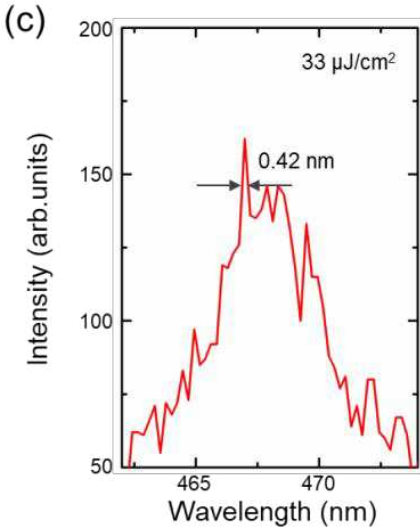

(e)

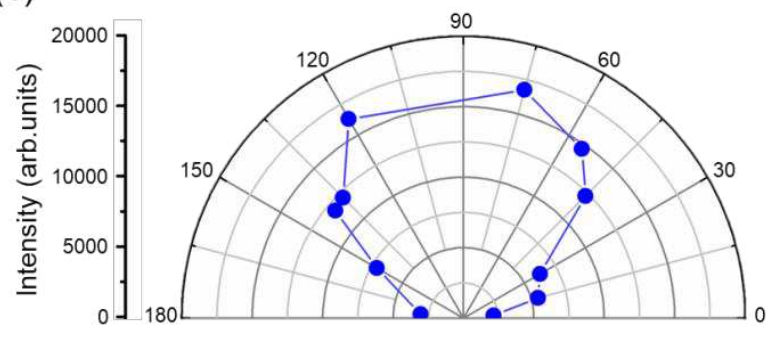

Figure 3 (a) Experimental setup of plasmonic random lasers on fiber facet. (b) Emission spectra of the plasmonic random system on fiber facet obtained under different pump power densities at the detection angle of $45^{\circ}$. (c) Specifics of emission spectra at pump power density of $33 \mu \mathrm{J} / \mathrm{cm}^{2}$, recorded by a high-resolution spectrometer with a resolution of $0.1 \mathrm{~nm}$. (d) Emission intensity of random lasing mode at $468 \mathrm{~nm}$ versus the pump energy density. Inset: corresponding images of the operating random laser on fiber facet. (e) The integrated intensity of the random laser as a function of the detection angle.

Refractive index sensing behavior is explored in terms of the change in emission performance of the plasmonic random laser on fiber facet. According to the laser principle, the laser mode is satisfied the equation ${ }^{44,45}$

$$
2 n L=m \lambda
$$

where $n$ is the effective refractive index of gain, $L$ is the resonant cavity length, $m$ is a positive integer mode number, and $\lambda$ is the lasing wavelength. Therefore, the media 
with different refractive index that exposed to the RL surface would affect the resonant mode by altering the effective refractive index of gain. Figure 4a summarizes the sensitivity to changes in the environmental refractive index by exposing different solutions to the RL surface. Six samples with the same parameter dip in solutions with different refractive index, such as methanol $(n=1.329)$, deionized water $(n=1.333)$, ethanol $(n=1.360)$, isopropyl alcohol $(n=1.379)$, glycol $(n=1.430)$, and oil $(n=1.475)$, respectively. Central wavelength shift of the laser emission is measured, which increases linearly with the refractive index (Figure 4a). And a refractive index sensitivity of $\Delta \lambda / \Delta n=1.24 \mathrm{~nm} / \mathrm{RIU}$ is estimated by linear fitting, where $\Delta n$ is the refractive index difference between environment and PFO. So, the plasmonic random laser on fiber facet can be used for refractive index sensor. Moreover, the near-surface sensitivity is characterized through detecting lasing wavelength variations caused by absorbing a monolayer of protein polymer Poly (Lys, Phe) (PPL, $1 \mathrm{mg} / \mathrm{ml}$ ). RL on the fiber facet is cultivated in the PPL solution and being coated with a single monolayer of PPL. During the self-assembled process, we monitored the wavelength at various cultivating stages of the polymeric monolayer ${ }^{14}$. Figure $4 \mathrm{~b}$ presents the temporal progression of the laser wavelength, which red-shifts rapidly after immersing in PPL solution for $4 \mathrm{~min}$. And then the changing rate is slowing down and tends to a definite value. The saturated phenomenon after $15 \mathrm{~min}$ is because the sensitivity decreases as the deposited layers of PPL on its surface increases. Therefore, the design of plasmonic random lasers fiber facet have high sensitivity to external environment refractive index and excellent near-surface sensitivity. 
(a)

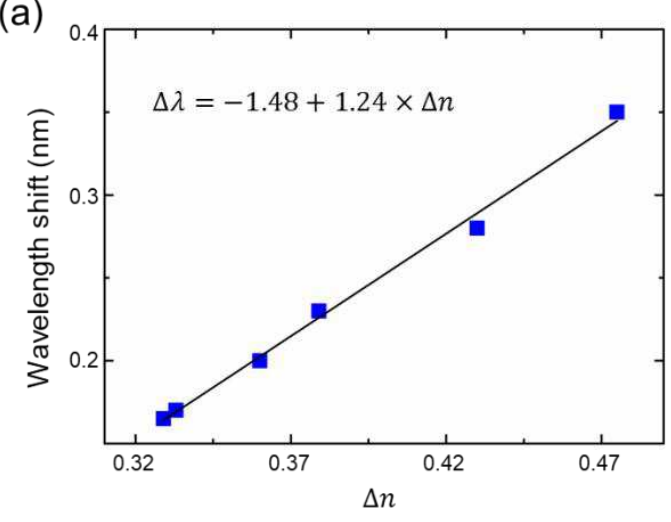

(b)

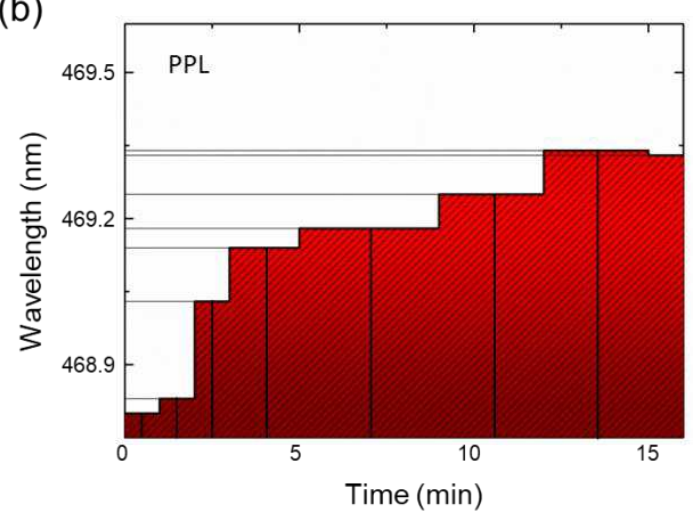

Figure 4 (a) The variation of wavelength shifts versus of external environment by exposing different solutions to the RL surface. (b) Dynamic detection of alternating deposition layers of single monolayer PPL.

The excellent surface sensing behavior of the random lasers makes it possible to detect biomolecules based on surface adsorption ${ }^{46}$. Figure 5a shows the sensing process for the RL on fiber facet based on a protein-protein interaction ${ }^{14}$. An initial baseline of emission wavelength is established before functionalizing the surface (the red line in Figure 5b). Protein A is firstly adsorbed to the surface of seven random lasers through noncovalent hydrophobic attachment for $20 \mathrm{~min}$ at room temperature. Then the fiber facet is subsequently rinsed by phosphate buffered saline (PBS). Corresponding antibody (human IgG) solutions with the concentrations of $34 \mu \mathrm{M}$ is exposed to the surface of RL decorated by Protein A. The human IgG molecules are specifically binding to Protein A by immunization interaction. The corresponding sensing kinetics for human IgG at $34 \mu \mathrm{M}$ with spectra measured every 5 min. As shown in Figure 5c, the wavelength red-shift tends to stable after 15 min, indicates that the sensing system reaches dynamic equilibrium during the binding process. The corresponding emission spectrum responsible for the random laser cultivated for 15 
min exhibits a shift of central wavelength about $0.53 \mathrm{~nm}$, as the blue line presented in Figure 5c. The phenomena demonstrate that plasmonic random lasers on fiber facet can be used to detect biomolecules, which is associated with the changes in effective refractive index. Another six RL sensors decorated by Protein A are exposed to human IgG solutions with six different concentrations $(3.4 \mu \mathrm{M}, 0.86 \mu \mathrm{M}, 0.34 \mu \mathrm{M}, 34$ $\mathrm{nM}, 3.4 \mathrm{nM}$, and $0.68 \mathrm{nM}$ ), respectively. The laser wavelength shifts behavior as a function of human IgG concentration is described in Figure 5d. The wavelength shifts at $43 \mu \mathrm{J} / \mathrm{cm}^{2}$ of representative sensors are $0.41 \mathrm{~nm}$ (for $3.4 \mu \mathrm{M}$ ), $0.29 \mathrm{~nm}$ (for 0.86 $\mu \mathrm{M}), 0.24 \mathrm{~nm}$ (for $0.34 \mu \mathrm{M}), 0.15 \mathrm{~nm}$ (for $34 \mathrm{nM}$ ), $0.11 \mathrm{~nm}$ (for $3.4 \mathrm{nM}$ ) and $0.09 \mathrm{~nm}$ (for $0.68 \mathrm{nM}$ ), respectively. As the binding sites of protein A on the surface are limited, sensing of human IgG with higher concentrations approaches saturation. And the detection limit of human IgG is $0.68 \mathrm{nM}$, which is comparable to other biosensors based on traditional resonance cavity ${ }^{14}$. And the results also demonstrate that RL-based biosensors have good sensing ability for the broad linear range between 84 $\mathrm{nM}$ and $12 \mu \mathrm{M}^{11,46}$. Herein, the sensing results indicate that random lasers provide a platform for developing random laser bio-probe on fiber facet for identifying virus or tracking drugs in human tissue, blood, and other body fluids. These random laser bio-probes with simple operation and high sensitivity have good application prospects in clinical medicine in the future. Moreover, these RL biosensors are easy prepared, low-cost and compact dimensions, which offers an opportunity for developing disposable biosensors like the traditional test paper. The one-off biosensors eliminate the complicated cleaning process and avoid the contaminating between different 
samples, demonstrating excellent potential of a disposal biomolecules "meter". These features are particularly crucial for reaching portable and integrated biomedical systems.

(a)

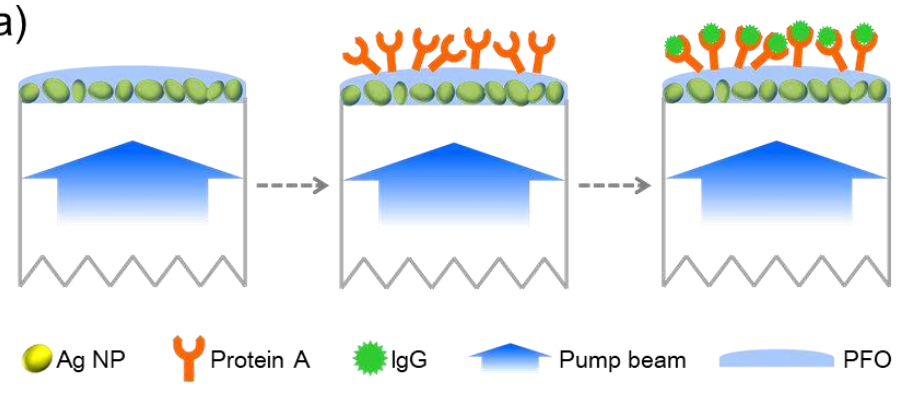

(b)

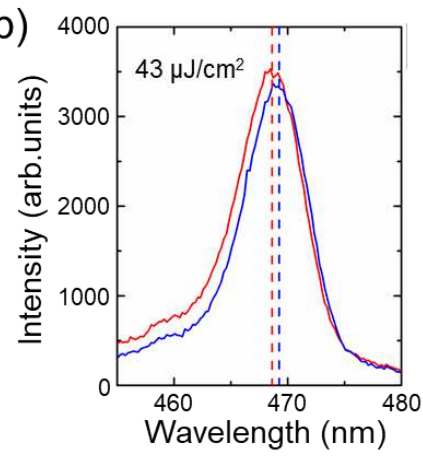

(c)

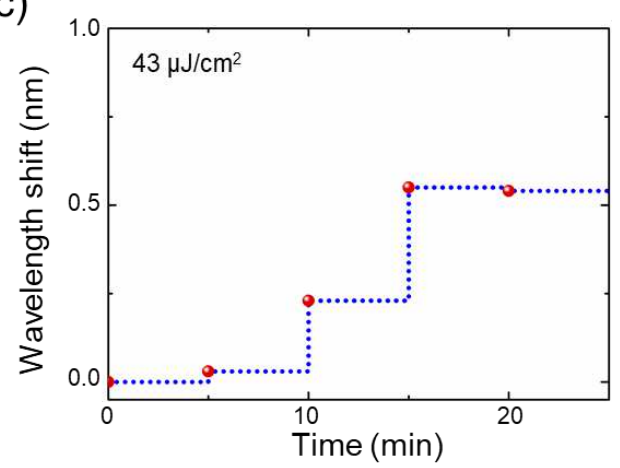

(d)

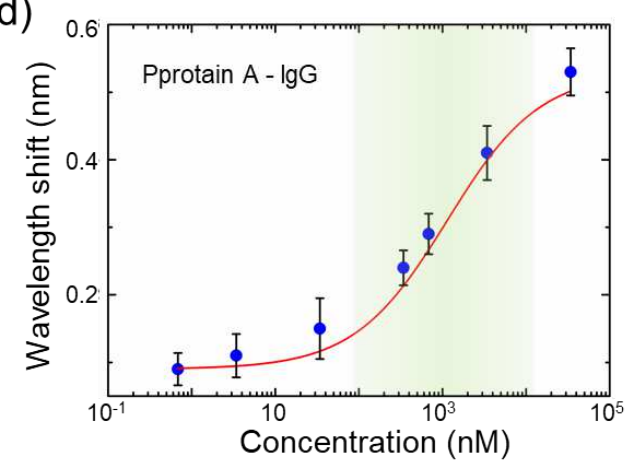

Figure 5 (a) Schematic diagram of capture immunoassay employed for $\lg G$ detection. (b)

Corresponding spectra of the RL-based sensor before functionalization with protein A (red line) and after being cultivated in $\operatorname{lgG}$ solution for $15 \mathrm{~min}$ (red line). (c) The temporal behavior of wavelength shift for the biosensors exposed to $\lg \mathrm{G}$ with the concentrations of $34 \mu \mathrm{M}$. (d) The variation of wavelengh shift as a function of $\operatorname{lgG}$ solution with different concentrations. Error bars represent the minimum value and maximum value of wavelength shift from the emission spectra acquired over 5 times.

\section{Conclusions}

In summery, a label-free RL biosensor on fiber facet is achieved based on protein-protein interaction. Plasmonic random scattering structure of Ag NPs is 
simply self-assembled on the facet of optical fiber by a hydrothermal method. The random laser on fiber facet is prepared by transplanting the PFO membrane upon the scattering structure, which operates with a low threshold of $30.5 \mu \mathrm{J} / \mathrm{cm}^{2}$ and weak directional selectivity. The random laser on fiber facet exhibits a linear relationship of the wavelength shift and changes in external refractive index near its surface. Near-surface sensitivity is characterized by adsorbing layers of PPL, demonstrating a satuated phenomena after $15 \mathrm{~min}$. The $\lg$ sensing process has been described in detail with the corresponding wavelength shifts of lasing mode though specific binding to protein-A. It is found that the RL biosensor have linear sensing curve for the broad linear range between $84 \mathrm{nM}$ and $12 \mu \mathrm{M}$ with a detection limit of $0.68 \mathrm{nM}$. It is believed that this work may promote the applications of plasmonic random lasers bio-probe in portable and integrated medical diagnostic platforms, provide fundamental understanding for the life science.

\section{Methods}

\section{Preparation of the plasmonic random laser on fiber facet}

Firstly, $\mathrm{AgNO}_{3}$ (Aldrich) is dissolved in DI water with a concentration of $0.2 \mathrm{~g} / \mathrm{ml}$. And dissolve $0.25 \mathrm{~g}$ of PVP ( $\mathrm{Mw}=1,300,000$; Aladdin) in DI water at $40{ }^{\circ} \mathrm{C}$. Secondly, the $\mathrm{AgNO}_{3}$ aqueous solution and PVP aqueous solution are mixed together as a homogeneous reaction solution. The optical fibers with a diameter of $800 \mu \mathrm{m}$ and length of $5 \mathrm{~cm}$ are ultrasonically cleaned for $5 \mathrm{~min}$ in ethanol and DI water to remove the absorbed contaminant. After the treatment, the polished facet of optical fiber is dipped in the prepared reaction solution. The fibers with reaction on their facets are 
heated at $200{ }^{\circ} \mathrm{C}$ for $0.5 \mathrm{~h}$ and then being cooled. Finally, PFO ( $\mathrm{Mw}=140,000$, American Dye Source) is dissolved in xylene with a concentration of $12.5 \mathrm{mg} / \mathrm{ml}$. The cooled fiber facet with Ag NPs is dipped in the solution of PFO, forming a PFO film with the thickness of $1 \mu \mathrm{m}$.

\section{Simulations}

The normalized electric field distribution of $\mathrm{Ag}$ NP is stimulated by the commercial software COMSOL. In simulations, a simplified model is made of an Ag particle with a diameter of $30 \mathrm{~nm}$. The excitation wavelengths are $466 \mathrm{~nm}$, corresponding to the central wavelength of random lasing. The permittivity of $\mathrm{Ag}$ is chosen from Johnson and Christy's experimental data ${ }^{1}$. The refraction index of the medium around Ag NP is 1.

To characterize the electric field enhancement of the random laser, we establish a calculation mode by putting five Ag particles with the diameter around $30 \mathrm{~nm}$ randomly distributed on the fiber, which is covered by a polymer film. The refraction index of the optical fiber and polymer are defined as 1.5 and 1.6, respectively. The excitation a plane wave polarized in the $x$ axis with a wavelength of $466 \mathrm{~nm}$, corresponding to the central wavelength of random lasing. The permittivity of $\mathrm{Ag}$ is chosen from Johnson and Christy's experimental data ${ }^{1}$.

\section{Optical measurements}

In our experiment, the fs pulses with a wavelength of $400 \mathrm{~nm}$ and repetition frequency of directly pump the end of optical fiber. The pump beam transferred along the optical fiber and excited the random laser film on the other end. The emission 
spectra are recorded by an optical fiber spectrometer (Ocean Optics, Maya Pro 2000, USA) with a spectral resolution of $1 \mathrm{~nm}$ and $0.1 \mathrm{~nm}$. The pumping source is a mode-locked laser (Coherent, Legend Elite, USA) with a wavelength of $400 \mathrm{~nm}$, pulse duration of $200 \mathrm{fs}$, output beam diameter of $2 \mathrm{~mm}$ and repetition rate of $1 \mathrm{kHz}$. A parameter $\theta$ is introduced to characterize the detected direction with respect to the orientation vertical to the fiber.

\section{Sensitivity measured of a Protein Monolayer}

Poly (Lys, Phe) (PPL, Sigma-Aldrich) is dissolved in DI water with concentration of $1 \mathrm{mg} / \mathrm{ml}$. After being rinsed by PBS ( $\mathrm{pH}=7.4)$ solution, the plasmonic random laser on fiber facet is exposed to the PPL solution. The PPL monolayer gradually self-assembled on the surface of RL. The emission spectra for different cultivating time are recorded. The wavelength shift response is saturated after $15 \mathrm{~nm}$, resulting to a sensitive thickness of $50 \mathrm{~nm}$.

\section{Sensing of $\operatorname{lgG}$}

First, seven biosensors based on plasmonic random laser on fiber facet with same parameters are rinsed by PBS ( $\mathrm{pH}=7.4)$. Next, protein $\mathrm{A}(\mathrm{Mw}=40 \mathrm{kDa}$, Sigma-Aldrich) is dissolved in PBS solution $(\mathrm{pH}=7.4)$ with a concentration of $0.5 \mathrm{mg} / \mathrm{ml}$. The six random lasers are cultivated in the solution for $20 \mathrm{~min}$. Then, the surfaces of random laser are rinsed with PBS solution three times to remove any protein A that not firmly attached. Lastly, the human $\operatorname{IgG}(\mathrm{Mw}=146 \mathrm{kDa}$, Sigma-Aldrich $)$ is cultivated in PBS solution $(\mathrm{pH}=7.4)$ with seven different concentrations of $0.68 \mathrm{nM}, 3.4 \mathrm{nM}, 34 \mathrm{nM}$, $0.34 \mu \mathrm{M}, 0.86 \mu \mathrm{M}, 3.4 \mu \mathrm{M}$ and $34 \mu \mathrm{M}$, respectively. The emission spectra of 
biosensor cultivated in the $\operatorname{IgG}$ solution at $34 \mu \mathrm{M}$ is recorded every $5 \mathrm{~min}$. And the other six biosensors in $\operatorname{lgG}$ solution are cultivated for $15 \mathrm{~min}$. Then the sensors are rinsed with PBS solution $(\mathrm{pH}=7.4)$ to remove unbound $\operatorname{lgG}$.

\section{Authors' contributions}

XS and TZ conceived and designed the work. XS, JT and KG performed the experimental work. XS and KG conducted the analysis of data. XS wrote the manuscript and $\mathrm{TZ}$ revised the manuscript. All authors read and approved the final manuscript.

\section{Funding}

The authors acknowledge the financial support of the National Natural Science Foundation of China (NSFC) (61822501, 11574015); Beijing Natural Science Foundation (Z180015). Beijing Postdoctoral Science Foundation (ZZ2019-126).

\section{Availability of data and materials}

All data generated or analyzed during this study are included in this published article.

\section{Ethics approval and consent to participate}

The study was approved by the Ethics Committee of Fourth Military Medical University.

\section{Consent for publication}

All the authors have approved the manuscript and agree with submission to this journal.

\section{Competing interests}

The authors declare that they have no competing interests.

\section{Author details}


College of Applied Sciences, Beijing University of Technology, Beijing, 100124, China

\section{References}

1. Lin M, Wang J, Zhou G, et al. Programmable engineering of a biosensing interface with tetrahedral DNA nanostructures for ultrasensitive DNA detection. Angew Chem Int Ed, 2015, 54: 2151-2155

2. Vollmer F and Arnold S. Whispering-gallery-mode biosensing: label-free detection down to single molecules. Nat methods, 2008, 5: 591-596

3. Chen Y C, Chen Q, and Fan X. Lasing in blood. Optica, 2016, 3: 809-815

4. Yang X, Shu W, Wang Y, et al. Turbidimetric inhibition immunoassay revisited to enhance its sensitivity via an optofluidic laser. Biosens Bioelectron, 2019, 131: 60-66

5. Hou M, Liang X, Zhang T, et al. DNA Melting analysis with optofluidic lasers based on fabry-perot microcavity. ACS Sens, 2018, 3: 1750-1755

6. Cai S, Han Z, Wang F, et al. Review on flexible photonics/electronics integrated devices and fabrication strategy. Sci. China Inform. Sci. 2018, 61, 060410

7. Campbell K, Stewart L D, Doucette G J, et al. Assessment of Specific Binding Proteins Suitable for the Detection of Paralytic Shellfish Poisons Using Optical Biosensor Technology. Anal Chem, 2007, 79: 5906-5914

8. Chen Q, Zhang X, Sun Y, et al. Highly sensitive fluorescent protein FRET detection using optofluidic lasers. Lab Chip, 2013, 13: 2679-2681

9. Mcconnell G, Mabbott S, Kanibolotsky A L, et al. Organic semiconductor laser platform for the detection of DNA by AgNP plasmonic enhancement. Langmuir, 2018, 34: 14766-14773

10. Sarkar D, Liu W, Xie X, et al. MoS2 Field-effect transistor for next-generation label-free biosensors. ACS Nano, 2014, 8: 3992-4003

11. Chiavaioli F, Gouveia C, Jorge P, et al. Towards a uniform metrological assessment of grating-based optical fiber sensors: From refractometers to biosensors. Biosensors, 2017, 7, 23-51

12. Chen Y C and Fan X. Biological lasers for biomedical applications. Adv Opt Mater, 2019, 7: 1900377

13. Gao Z, Zhang W, Yan Y, et al. Proton-controlled organic microlaser switch. ACS Nano, 2018, 12: 5734-5740

14. Lu M, Choi S S, Irfan U, and Cunningham B T. Plastic distributed feedback laser biosensor. Appl Phys Lett, 2008, 93: 111113

15. Retolaza A, Martinez-Perdiguero J, Merino S, et al. Organic distributed feedback laser for label-free biosensing of ErbB2 protein biomarker. Sens Actuators B Chem, 2016, 223: 261-265

16. Watanabe K, Nomoto M, Nakamura F, et al. Label-free and spectral-analysis-free detection of neuropsychiatric disease biomarkers using an ion-sensitive GaInAsP nanolaser biosensor. Biosens Bioelectron, 2018, 117: 161-167

17. Watanabe T, Saijo Y, Hasegawa Y, et al. Ion-sensitive photonic-crystal nanolaser sensors. Opt Express, 2017, 25: 24469-24479

18. Yang A, Hoang T B, Dridi M, et al. Real-time tunable lasing from plasmonic nanocavity arrays. 
Nat Commun, 2015, 6: 6939

19. Cao H, Zhao Y G, Ho S T, et al. 17. Phys Rev Lett, 1999, 82: 2278-2281

20. Zhai T, Zhang X, Pang Z, et al. Random laser based on waveguided plasmonic gain channels. Nano Lett, 2011, 11: 4295-4298

21. El-Dardiry R G S and Lagendijk A. Tuning random lasers by engineered absorption. Appl Phys Lett, 2011, 98: 161106

22. Zhai T, Xu Z, Li S, and Zhang X. Red-green-blue plasmonic random laser. Opt. Express, 2017, 25 : 2100-2016

23. Popov O, Zilbershtein A, and Davidov D. Random lasing from dye-gold nanoparticles in polymer films: Enhanced gain at the surface-plasmon-resonance wavelength. Appl Phys Lett, 2006, 89: 191116

24. Abaie B, Mobini E, Karbasi S, et al. Random lasing in an Anderson localizing optical fiber. Light: Sci Appl, 2017, 6: e17041

25. Mujumdar S, Cavalieri S, and Wiersma D S. Temperature-tunable random lasing: numerical calculations and experiments. J Opt Soc Am B, 2004, 21: 201-207

26. Gaio M, Caixeiro S, Marelli B, et al. Gain-Based Mechanism for pH Sensing Based on Random Lasing. Phys Rev Applied, 2017, 7:034005

27. Polson R C and Vardeny Z V. Random lasing in human tissues. Appl Phys Lett, 2004, 85: 1289-1291

28. Yin L, Cheng H, Mao S, et al. Dissolvable Metals for Transient Electronics. Adv Funct Mater, 2014, 24: 645-658

29. Shinohara S, Fukushima T, and Harayama T. Light emission patterns from stadium-shaped semiconductor microcavity lasers. Phys Rev A, 2008, 77: 033807

30. Ignesti E, Tommasi F, Fini L, et al. A new class of optical sensors: a random laser based device. Sci Rep, 2016, 6: 35225

31. Dice G D, Mujumdar S, and Elezzabi A Y. Plasmonically enhanced diffusive and subdiffusive metal nanoparticle-dye random laser. Appl Phys Lett, 2005, 86: 131105

32. Meng X, Fujita K, Murai S, et al. Plasmonically controlled lasing resonance with metallic-dielectric core-shell nanoparticles. Nano Lett, 2011, 11: 1374-1378

33. Ziegler J, Wörister C, Vidal C, et al. Plasmonic nanostars as efficient broadband scatterers for random lasing. ACS Photonics, 2016, 3: 919-923

34. Chou C H, Yang C H, Hsu C S, and Chen T M. Hybrid white-light emitting-LED based on Luminescent Polyfluorene Polymer and Quantum Dots. J Nanosci Nanotechnol, 2007, 7: 2785-2789

35. Zhang H, Lu Q, Zuo F, et al. A sandwich electrochemiluminescent biosensor based on enzymatic reaction to in situ generate coreactant of conjugated polymer for Con A detection. Sensors and Actuators B, 2017, 241: 887-894

36. Wu C and Chiu D T. Highly fluorescent semiconducting polymer dots for biology and medicine. Angewandte Chemie, 2013, 52: 3086-3109

37. Villemin E, Gravel E, Izard N, et al. Polymer-decorated carbon nanotubes as transducers for label-free photonic biosensors. Chem Eur J, 2015, 21: 18649-18653

38. Shi X, Wang Y, Wang Z, et al. Random lasing with a high quality factor over the whole visible range based on cascade energy transfer. Adv Opt Mater, 2014, 2: 88-93

39. Fu Y, Zhang J, and Lakowicz J R. Largely enhanced single-molecule fluorescence in plasmonic 
nanogaps formed by hybrid silver nanostructures. Langmuir, 2013, 29: 2731-2738

40. Punj D, Regmi R, Devilez A, et al. Self-assembled nanoparticle dimer antennas for plasmonic-enhanced single-molecule fluorescence detection at micromolar concentrations. ACS Photonics, 2015, 2: 1099-1107

41. Flauraud V, Regmi R, Winkler P M, et al. In-plane plasmonic antenna arrays with surface nanogaps for giant fluorescence enhancement. Nano Lett, 2017, 17: 1703-1710

42. Khatua S, Paulo P M R, Yuan H, et al. Resonant plasmonic enhancement of single-molecule fluorescence by individual gold nanorods. ACS Nano, 2014, 8: 4440-4449

43. Li S, Wang L, Zhai T, et al. Plasmonic random laser on the fiber facet. Opt Express, 2015, 23 : 23985-23991

44. Chen Y L, Chen C L, Lin H Y, et al. Enhancement of random lasing based on the composite consisting of nanospheres embedded in nanorods template. Opt Express, 2009, 17: 12706-12713

45. Nathan M I, Fowler A B, and Burns G. Oscillations in GaAs spontaneous emission in Fabry-Perot cavities. Phys Rev Lett, 1963, 11: 152-154

46. Chiavaioli F, Trono C, Giannetti A, et al. Characterisation of a label-free biosensor based on long period grating. J. Biophoton., 2014, 7: 312-322

47. Johnson P B and Christy R W. Optical constants of the noble metals. Phys Rev B, 1972, 6: 4370-4379 


\section{Figures}

(a)

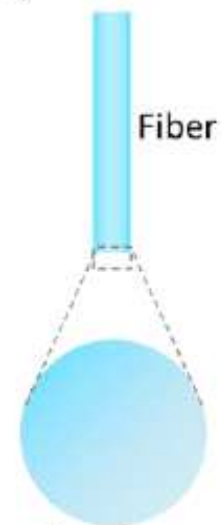

Fiber facet

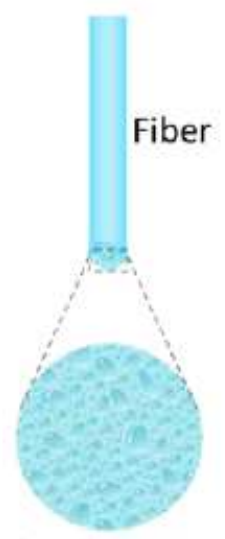

$\mathrm{AgNO}_{3}+\mathrm{PVP}$

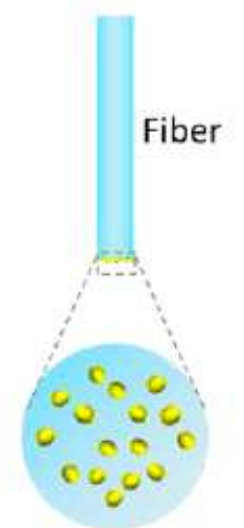

Ag NPs

(c)

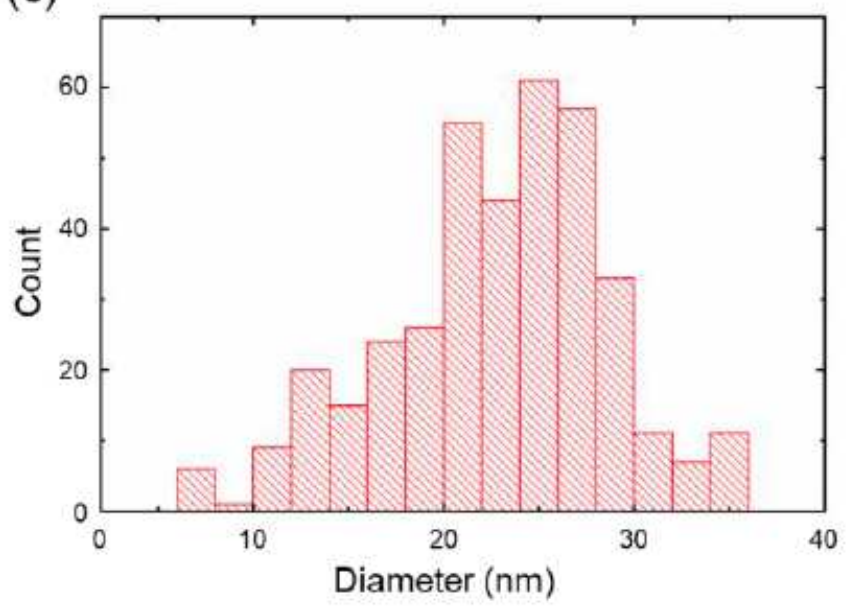

(b)

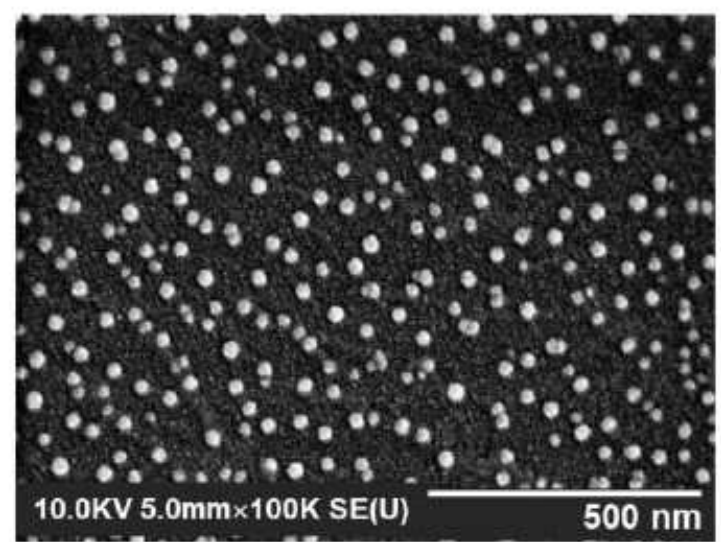

(d)

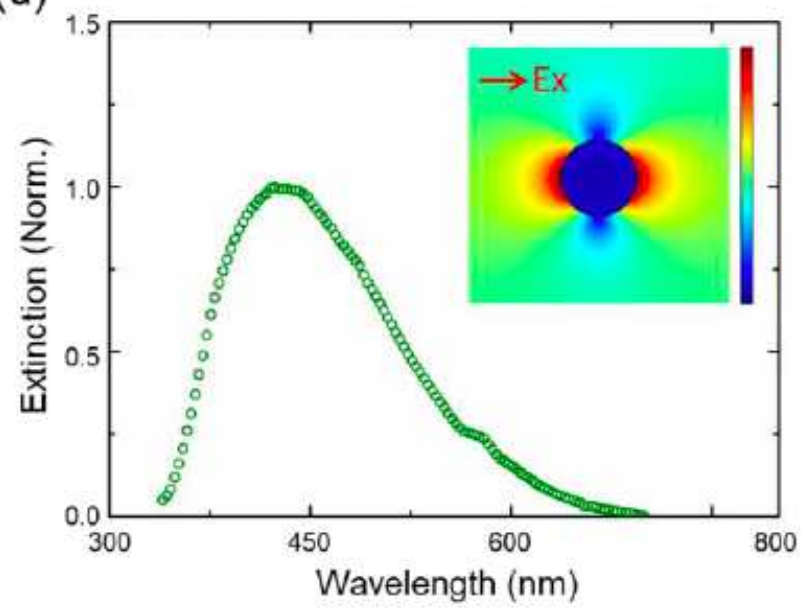

\section{Figure 1}

Figure 1 (a) Schematic illustration of the synthesis process for scattering structure on fiber facet (b) Scanning electron microscopy (SEM) image of Ag N P s self assembl ed on fiber facet. (c) Statistical distribution of Ag N P s that self assembl ed on fiber facet d) The extinction spectrum of Ag N P s . Inset: the normalized electric field intensity of $\mathrm{Ag} \mathrm{N} \mathrm{P}$, the excitation is a plane wave polarized in the $\mathrm{x}$ axis with a wavelength of $466 \mathrm{~nm}$. 
(a)

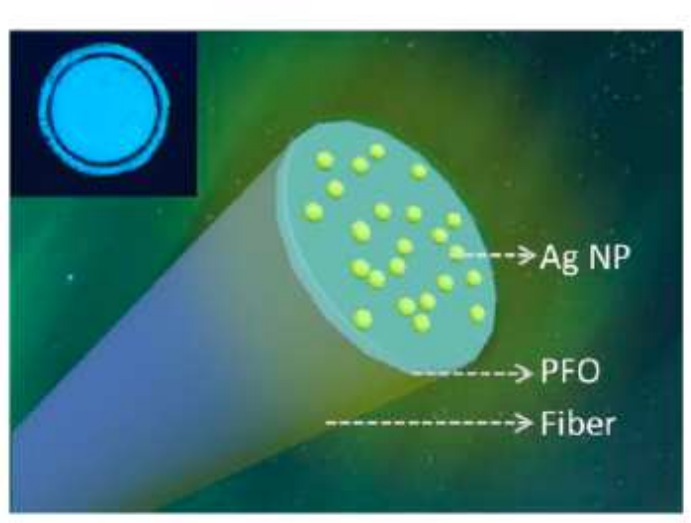

(c)

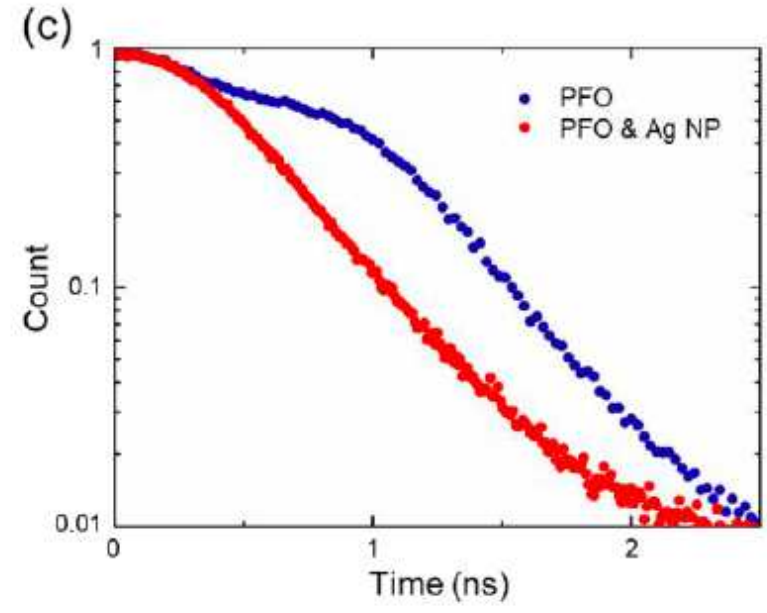

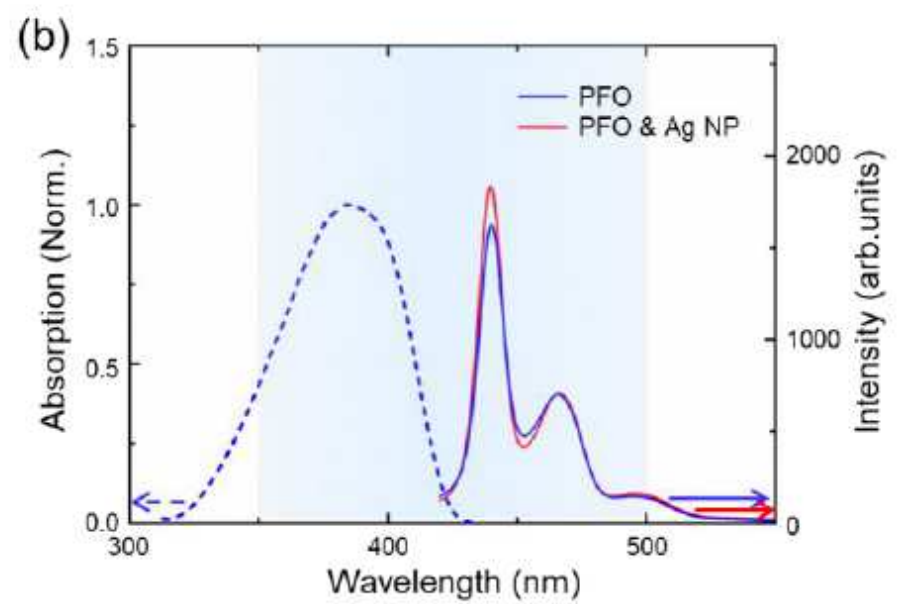

(d)

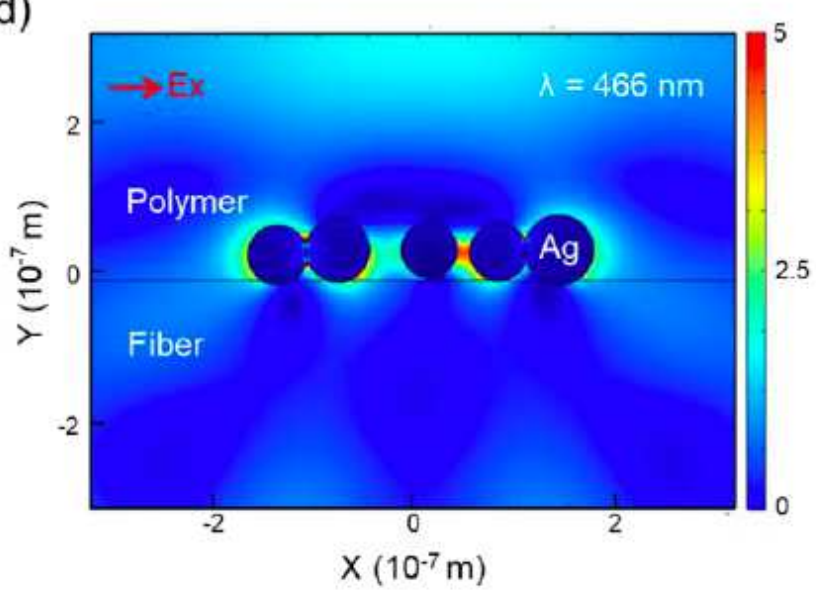

Figure 2

(a) Design of the plasmonic random laser on fiber facet (b) Absorption spectra ( blue dash) and $p$ hotoluminescence spectra of PFO blue solid )), p hotoluminescence spectra of polymer with Ag N P s red solid) ( The PL lifetime of PFO with (red dots) and without Ag N P s (blue dots) (d) The normalized electric field intensity of Ag N P s on the fiber facet covered by PFO, the excitation is a plane wave polarized in the $x$ axis with a wavelength of $466 \mathrm{~nm}$. 
(a)

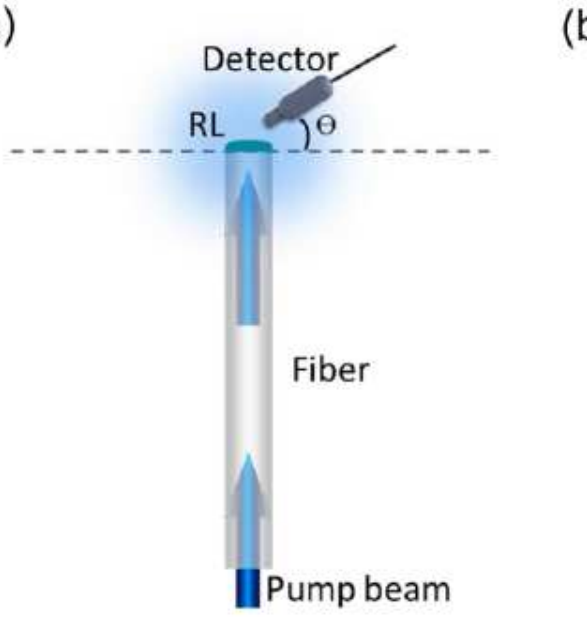

(d)

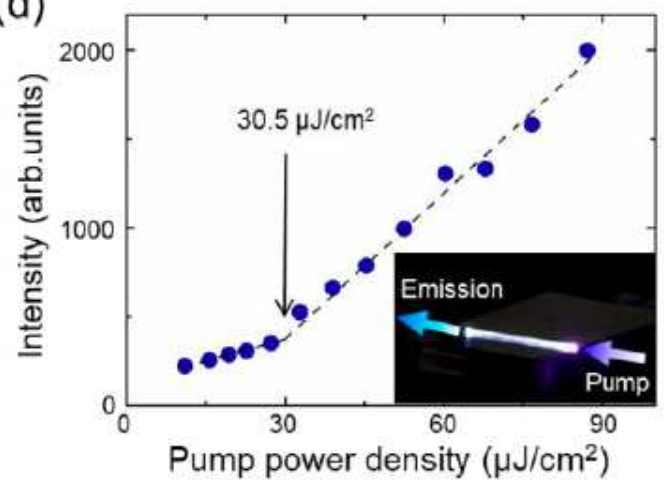

(b)

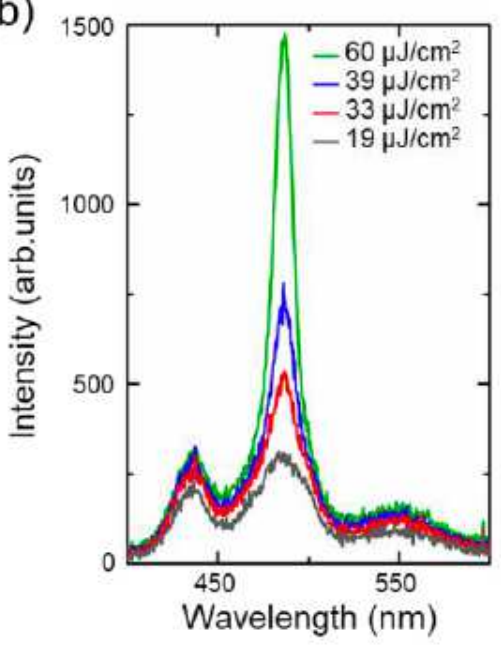

(c)

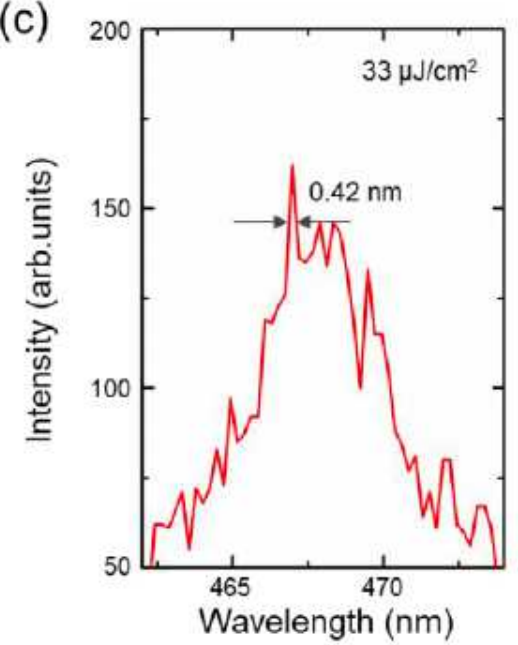

(e)

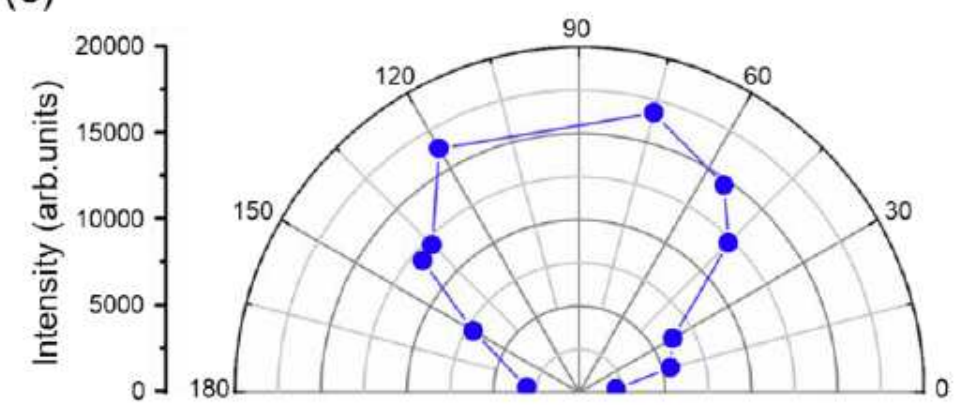

Figure 3

(a) Experimental setup of plasmonic random lasers on fiber facet . (Emission spectra of the plasmonic random system on fiber facet obtained under different pump power densities at the detection angle of 45 ${ }^{\circ}$. ( Specifics of emission spectra at pump power densit y of $33 \mu \mathrm{J} / \mathrm{cm} 2$, recorded by a high-resolution spectrometer with a resolution of $0.1 \mathrm{~nm}$ (Emission intensity of random lasing mode at $468 \mathrm{~nm}$ versus the pump energy density Inset: corresponding images of the operating random laser on fiber facet . (e) The integrated intensity of the random laser as a function of the detection angl $e$ 
(a)

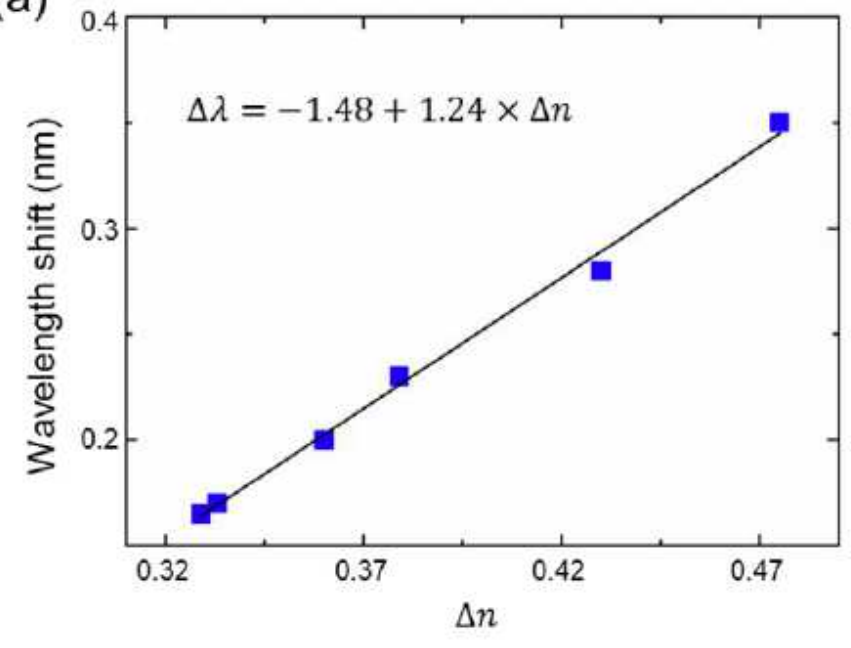

(b)

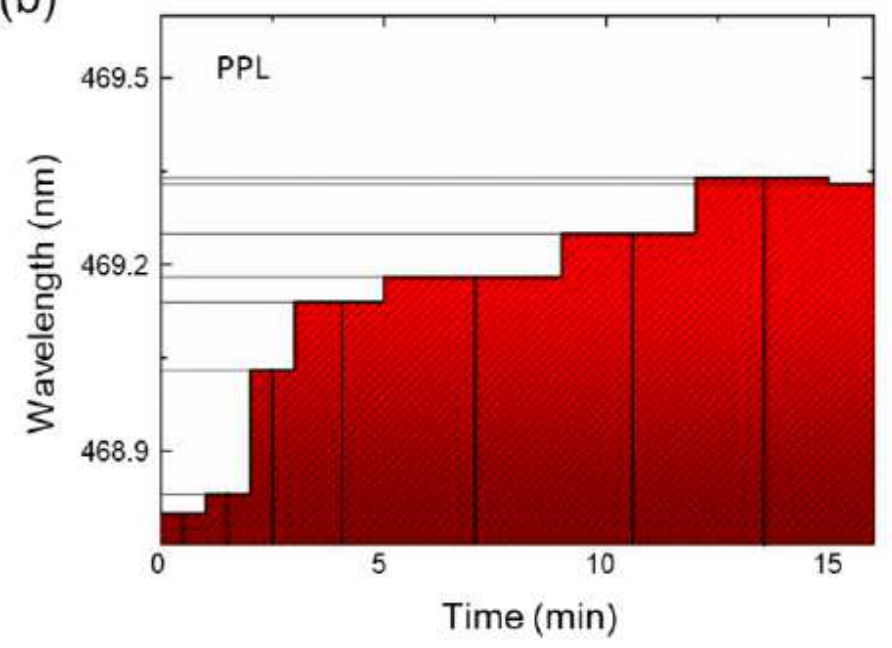

\section{Figure 4}

(a) The variation of wavelength shifts versus of external environment by exposing different solutions to the RL surface surface. (b) Dynamic detection of alternating deposition layers of single monolayer PPL. 
(a)

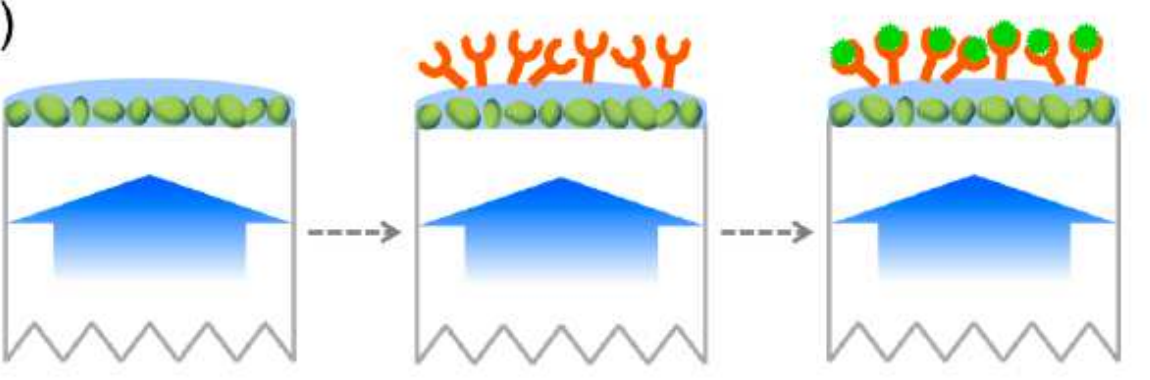

Ag NP Yrotein A the (b)

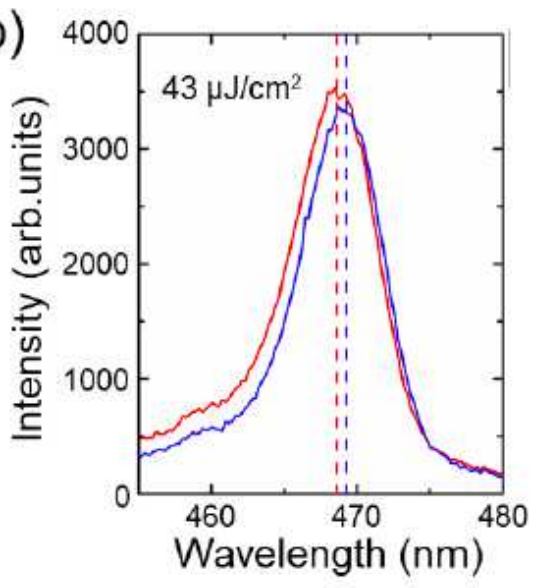

(c)

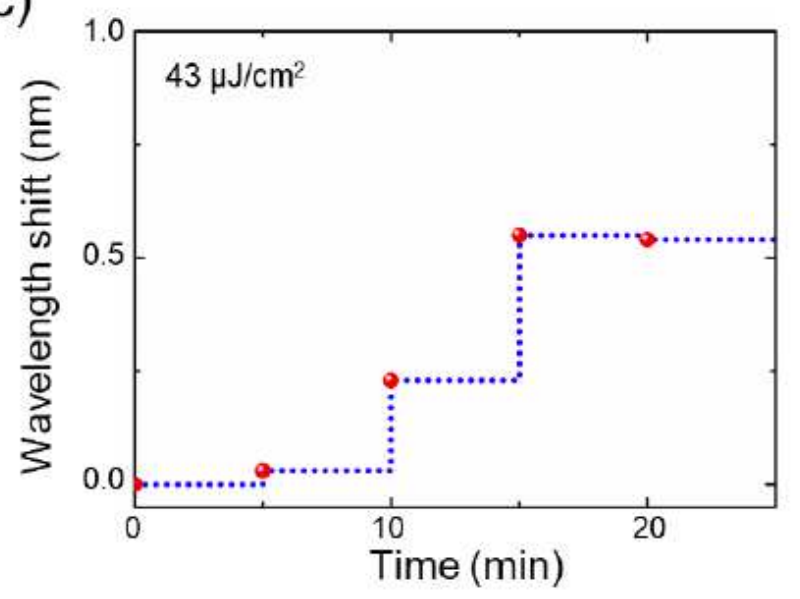

(d)

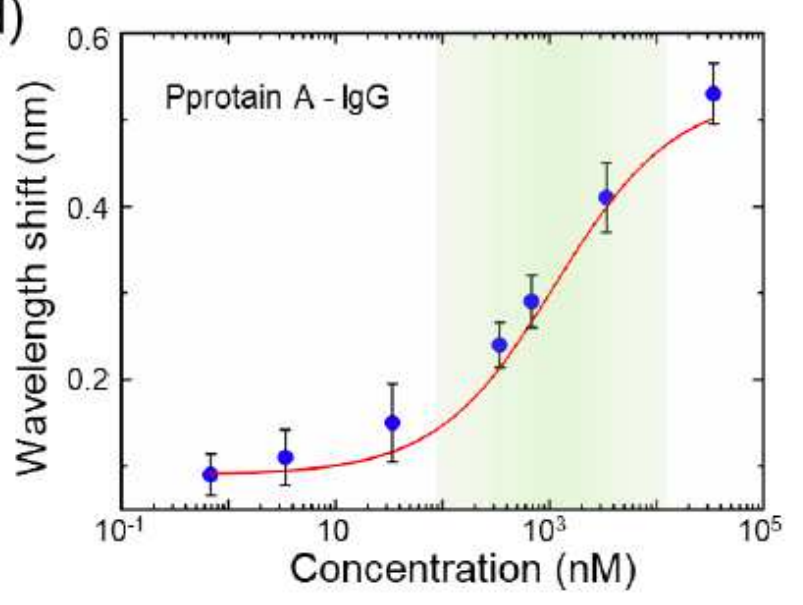

Figure 5

Figure 5 (a) Schematic diagram of capture immunoassay employed for IgG detection ( Corresponding spectra of the RL based sensor before functionalization with protein A (red line) and after being cultivate $\mathrm{d}$ in IgG solution for $15 \mathrm{~min}$ (red c) The t emporal behavior of wavelength shift for the biosensors exposed to $\lg G$ with the concentrations of $34 \mu \mathrm{M}$ (d) The variation of wavelengh shift as a function of IgG solution with different concentrations. Error bars represent the minimum value and maximum value of wavelength shift from the emission spectra acquired over 5 times. 